\title{
INTERNET INTELLIGENT SERVICES OF VEHICLES BASED ON THE INTERNET OF THINGS AND MULTI- AGENT SYSTEMS
}

\author{
Ashraf Darwish* and Cristina Turcu** \\ * Helwan University, Faculty of Science, Cairo, Egypt \\ ashraf.darwish.eg@ieee.org \\ ** Stefan cel Mare University of Suceava, Faculty of Electrical Engineering and Computer Science, Romania \\ cristina@eed.usv.ro
}

Received 17/1/2018 Revised Accepted 24/3/2018

\begin{abstract}
The facilities offered by the distributed computing systems such as Internet of Things (IoT), multi-agent systems (MASs) and vehicular networks can be used to integrate such paradigms to provide several services of Internet of Vehicles (IoV). The core objective of this paper is to present the integration of Multi-agent Systems (MASs) with IoT and IoV as a new model in Cypher Physical System (CPS) of smart vehicles application. MASs are more effective when we employ them with distributed computing infrastructures as with IoV to achieve more high performance. On the other hand, there is a lack of research works and a few papers in the literature till today with the integration of such paradigms, MAS, IoT, and IoV. The features of the proposed integration are based on the main characteristics of intelligent agents like intelligence, flexibility, proactivity and reactivity, and autonomy which can be employed in IoV paradigm to provide new solutions and intelligent services that will explore new advances and different facilities in transportation problems. Also, IoT provides an adequate environment for communication with MASs paradigms and applications as it provides reliable, flexible, effective, and smart services to vehicular networks. Moreover, integrating MASs with IoT and IoV could provide flexible, autonomous, and intelligent services of IoV. Some limitations and challenges of the proposed integration method in this paper are presented with some future research trends.
\end{abstract}

Keywords: Intelligent agent, Multi-intelligent agents, Internet of Things (IoT), Internet of Vehicles (IoV), Smart devices.

\section{Mathematics Subject Classification: 68, 93, 94}

\section{Introduction and Related Work}

In the last decade, there is an increasing interest in using vehicular networks [1], [2]. With the increasingly powerful communications, sensing, data processing and enabling IoT technology can be used to enhance vehicles capabilities, such as utilizing resources among vehicles on the roads or in parking space. Also, IoT can be employed to track the movement of vehicles and predicting their location. The recent advances in smart devices (e.g., sensors) and vehicles electronic control have been providing better communication and computing power, facilitating, for example, the communications between vehicles. The main feature of IoT paradigm depends on its infrastructure that can facilitate and allow the interconnections and communication between different things/objects and other entities, such as human (drivers, passengers), wireless sensor networks, transportation devices, and cell phones which can be used in real-time monitoring. But the capability of IoT devices to sense and process data from their environment could be enhanced through added intelligence and reasoning features. Intelligent agent systems have developed these characteristics so the combination of both paradigms, IoT and intelligent agent (IAs), by modeling intelligent agents in IoT devices is a very promising approach that will enable a more powerful of IoT capabilities. But, instead of proposing new applications, integrating existing applications through agents is a promising direction. However, this integration addresses some critical issues and challenges that can affect all network communications, such as privacy security which especially given the sensitivity of the information exchanged by agents through IoT. The following Figure 1 explores the development of IoT technology in the last years such as network, software and hardware, and algorithms and data processing for various applications such as vehicular networks. 

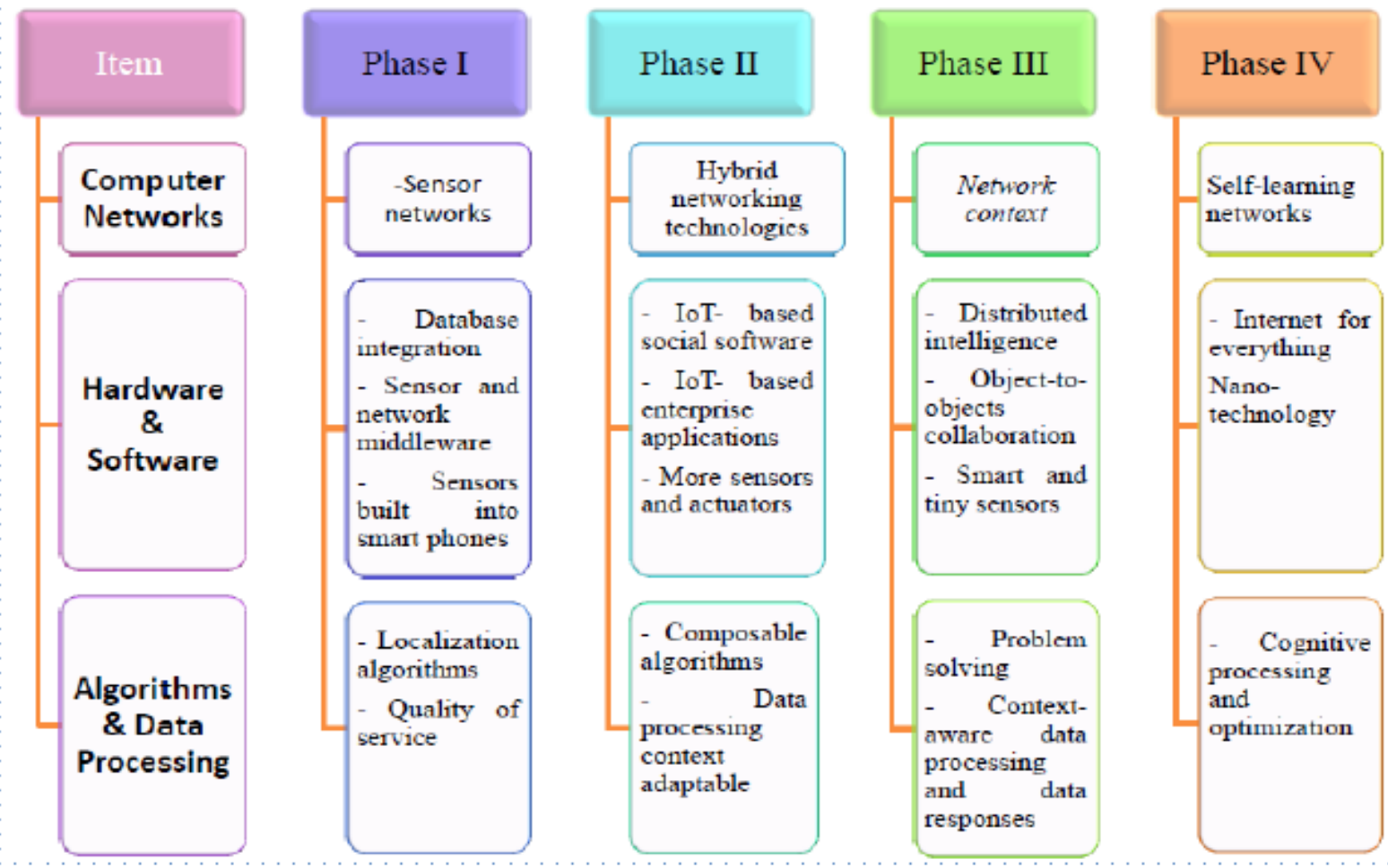

Fig.1. The development stages of IoT

Internet of Vehicles (IoV) has been adopted thanks to the advancements of IoT. The core objective of IoV is intelligently integrated multiple users, vehicles, things and devices, and networks to provide solutions to the transportation problems to be manageable, controllable, and operational. Such a network system that could provide services for transportation in the smart cities is still under investigation and application. However, it is highly desirable for the future of transportation. In order to deal with the complex problems and limitation of IoV (large scale, physical distribution, decentralised control, resource boundedness, information uncertainty, adaptiveness, etc.), we propose in this paper a new method that depends on the features of the ABC (agent-based computing) paradigm $[3,4]$, which is focusing on the intelligent agent described as entity that is proactive and autonomous and fulfill various purpose. The paradigm of $\mathrm{ABC}$ can model distributed software systems based on MASs. In this scenario, agents can be considered as entities of networked software which can achieve various specific goals for a user and have a degree of intelligence to allow them to perform their tasks, to interact with each other's and with their environment. Intelligent agents have been effectively used in some areas of applications to build dynamic and robust distributed systems. For these reasons, we claim in this paper focusing on features and characteristics of IoV systems and its environment to integrate it with MASs $[5,6]$ which will be explained in the next section.

To the best of our knowledge, there are a few types of research in the literature in the topic of this paper which describe different strategies to develop and manage agent-oriented IoV systems. Also, the researchers of IoV is in increasing in the last years. Therefore, authors of this paper conducted an extensive review of the literature with the related researches in that area from different well known five main academic databases (Web of Knowledge, ACM digital library, IEEE Xplore, INSPEC, and Science Direct) to provide awareness and understanding of the state-of-art and explore the future opportunities of research in the adoption of agents in IoV. Therefore, in this paper, we focus on identifying the opportunities and limitations of MASs in the IoV and identifying the future trends of IoV in smart cities. For instance, we found that $76 \mathrm{IoV}$-related journal and conference articles in Web of Knowledge that had been published since 2011. Figure 2 explores the related articles that are stored in the Web of Knowledge database from 2011 to 2016. The figure describes the increasing number of research on IoV in the last five years. 


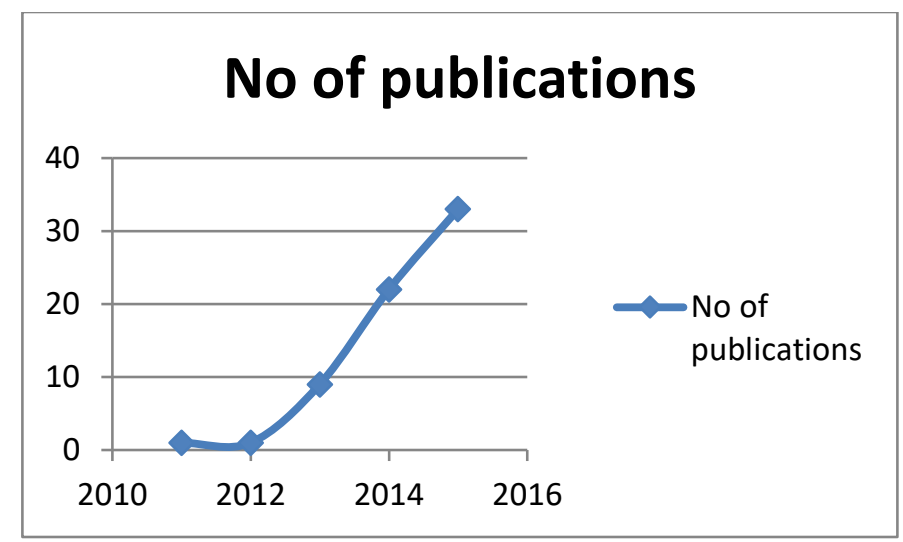

Fig. 2. The number of IoV publications by year in Web of Knowledge

Also, we found 69 Internet of Vehicles and 18 Internet of Vehicle-related journal and conference works published since 2010 to 2016 by searching the IEEE database. The trend for this database is similar to Web of the Knowledge database. Some of these publications reveal various advantages and problems in IoV. Multi-agent technologies provide new methods for solving certain of these problems. But, this topic is still in its infancy.

The rest of this paper is organized as follows. Section II describes the main concepts and features of MASs briefly. Section III presents the integrated model of MASs and IoV and explores the MASs based IoV. Section IV explores some applications. Section $\mathrm{V}$ presents the limitation, challenges, and future work of this integration. Section VI concludes this paper.

\section{Basics and Background}

To the best of our knowledge, there are no attempts at integrating MASs with the IoV, but the software agents are used to increase the performance of the IoT. Thus, intelligent agents can enhance the services that the IoT can offer to its users. The five-layer architecture of IoT is presented in Figure 3. For example, the user centric agents can be used to enable the automation of user queries. Figure 3 shows the basic architecture of IoT which consists of three basic layers:

- Network layer: this layer is the most important layer in the IoT architecture. The function of this layer is to address all the objects and is responsible for transmitting the secure information between the other two layers as in OSI network model. Some protocols of communication and transmission of data are part of this network layer such as Bluetooth, ZigBee, and WiFi.

- Application layer: this layer is responsible for carrying commands from applications to the end-device actuators

- Perception layer: this layer is responsible for collecting information and identifying objects and includes some key technologies such as RFID, sensors, nanotechnology, and barcodes.

Recently, there is a five-layer architecture that has been introduced in $[7,8]$ by adding two layers as in Figure 3:

- Processing layer (or middleware layer): this layer is responsible for data manipulation which can be collected by objects over the network (store, analyze, and process). Thus, database software, intelligent processing, and ubiquitous computing are employed in this layer

- Business layer. The layer has some different functions (a) it manages the IoT system and its applications and services; (b) it receives data from the application layer to build models of business, flowcharts, and graphs (c) it provides privacy of users. 


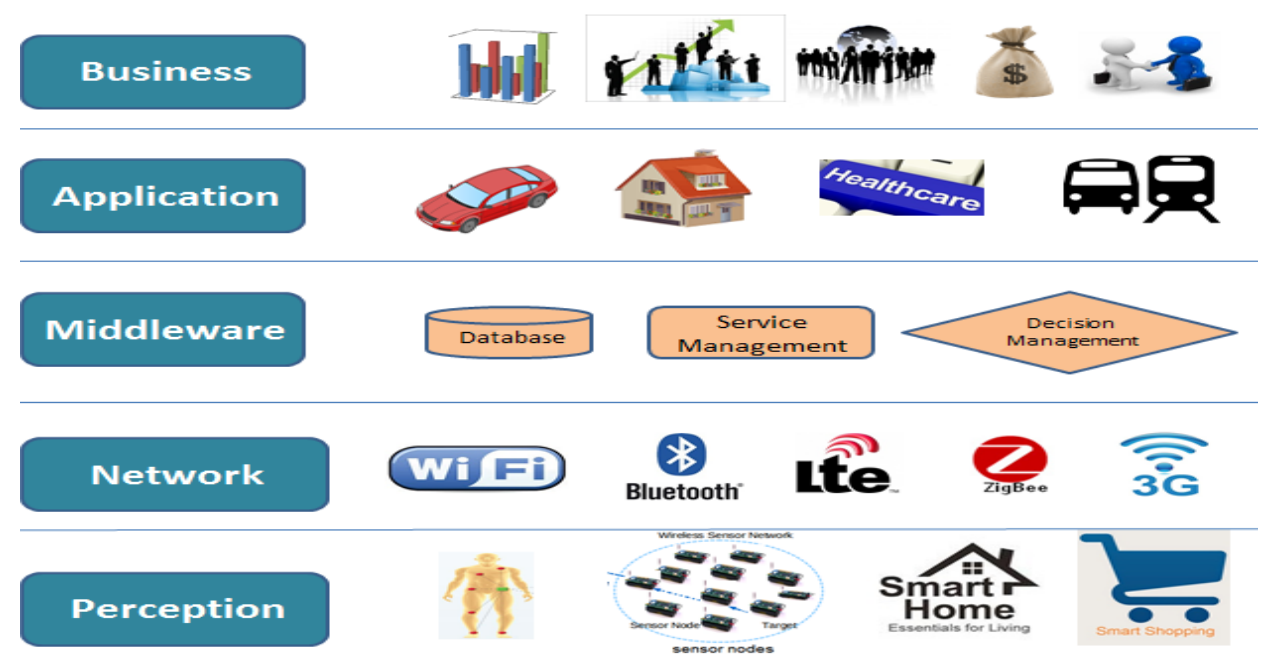

Fig. 3. IoT layer Architecture [7, 8]

A. Multi-agent systems

The intelligent agent can be described as computational entity or software programs which can behave with a degree of independence to perform actions that are needed to solve a given problem. To solve complex problems of IoV, we consider that an agent-based solution is sustained by the following characteristics of the agents:

1. Autonomy. Agent operates autonomously without control over its actions or the internal state, and it can modify the used methods to achieve its goals.

2. Reactivity and Proactivity: Agents can respond to changes in the external environment and consequently adapt their behavior by planning to make their decisions based on the internal decisions to achieve their goals.

3. Cooperation and Communication: Agents can communicate and make interaction with other agents in a system of multiple agents to receive instructions and provide responses, exchange information, and interact to achieve their goals.

4. Agents Negotiation: Agents can organize conversations with other agents to the cooperation between them.

5. Agent's Knowledge. The ability of how the agent's actions affect its environment to improve the decision-making process and increase its performance over time to perform reactivity and proactivity.

MAS paradigm is composed of agents as entities that can act in a collection of agents to perform specific tasks. This means that agents are difficult to exist and operate only in isolation, they can interact with other agents regardless they are artificial or real agents in its environment. According to [9] the following main features of MASs require that agents should integrate with IoT:

1. Each agent has a limited capability of information on the global task to be performed for solving a problem.

2. Data in MASs are decentralized computing paradigms with no global control system.

3. The computational capability is asynchronous.

Due to distributed computing systems are decentralized paradigms, MASs share several characteristics with other distributed systems, sensor networks, objects, IoT, etc. Also, MASs provide some features that provide advantages of them than other distributed computing models.

\section{The Integration of IoV and Multi-agent Systems}

IoV network is a new technology that can support the communication channels for interactions between vehicles and other things or devices. This paper proposes the integration between IoV and MASs to provide efficient solutions for transportation which can be represented in vehicle-to-vehicle (V2V) communications and vehicle-to-infrastructure (V2I). There are different communication standards such as the IEEE 802.15.4 standard that can be used in the IoV as in Figure 4.

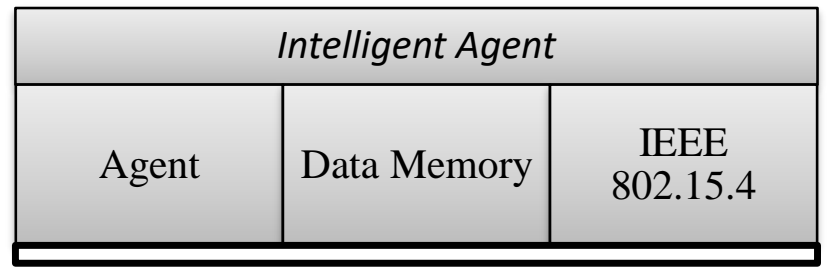

Fig. 4. Intelligent Agent

This standard is an efficient and candidate to IoV which can comprise energy-efficient medium access control and physical layer. Moreover, vehicular network as wireless connectivity-enabled vehicles enables them to communicate with their environments (internal/external). Connected vehicles represent the building blocks of IoV, which is a dynamic mobile communication system that can be used in sharing, gathering, processing, and computing with security requirements of information which will enhance the evolution to the next generation of intelligent transportation. Nowadays, monitoring the traffic congestion in the smart cities 
can be achieved by urban IoT technology. The services of the smart cities that can be enabled by urban IoT depend on monitoring the traffic congestion in the city. Sensing capabilities of IoT monitoring with GPS installed on modern vehicles can be employed in traffic by using the smart

sensors that collect data from different sources and store and analyze it to provide the information that is important for the decision makers of the city and citizens. Nowadays, there is an increasing need to modern vehicles which are equipped with intelligent sensors, such as sensors for detecting driver's fatigue and road conditions and, sensors for monitoring water temperature in the cooling system and tire pressure, and other sensors for autonomous control. IoT is a paradigm in which numerous devices (and not only) connect to the Internet and communicate with other networks using different protocols and standards. These devices may include sensors, RFID, laptops, desktops, servers, vehicles, etc.

$\mathrm{IoV}$ is a new concept refers to the interconnection of vehicles and with other devices. IoV is usually used for providing communication amongst vehicles to support various applications ranging from data transmission for traffic safety to information services. In this scenario, vehicles act as intelligent machines having communication, sensing and storage capabilities. Due to the availability of advanced hardware and software in IoT resources nowadays, vehicular networks have gained an interest for some governments all over the world. However, there are some shortages in IoT technology which are related to the lack theories, the architecture of technology, and standardization to integrate the both real and virtual worlds in a unified framework [10]. IoV, as described in Figure 5, is used to provide communication amongst interconnected vehicles to support various services such as data transmission for traffic safety and information services.

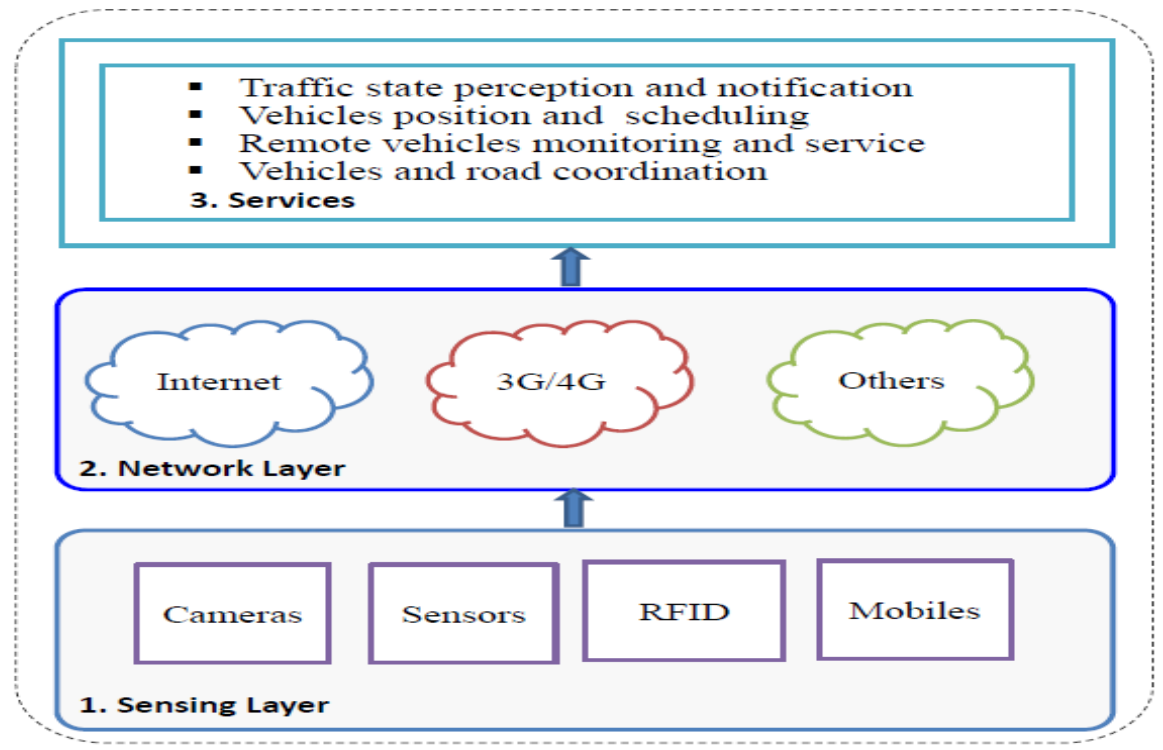

Fig.5. The Internet of Vehicles Model

In a unified model, IoV and intelligent agents must coordinate their activities to achieve various wide goals and tasks. IoV technology is classified by its technology, distributed control, ambiguity, and multiple stakeholders with several objectives and limited resources. All these characteristics make IoV suitable as an application domain to integrate with MAS. On the other hand, as mentioned before, MAS is composed of autonomous, reactive and proactive agents that can act, sense and interact with each other's to achieve its goals. Also, these agents can use the available information to be flexible in making its decisions. Such challenge represents the main research problems of MAS. Moreover, there are some features that are required by IoV to make the use of this technology a reality:

1. Physical distribution. IoV involves information and computational resources that are physically distributed as in the case of vehicles.

2. Resources limitation. In IoV, there are some boundaries and limitations such as computational capability, energy and communication resources. Moreover, vehicles usually located in rapid changing environments which exhibit capabilities to cope with time constraints. However, vehicles must be able to operate with their computation capabilities which are low with slow resources and communication power.

3. The uncertainty of information. Usually, there is a lack of information regarding the states of vehicles, the environment where the vehicle is situated, and the effects of vehicles' actions.

4. Large scale. IoV consists of a large number of components, such as vehicles, which cause large-scale computational approaches.

5. Decentralized control. Vehicles need to interact to achieve their tasks that cannot be possible by the operation of a single vehicle. But, such interaction must be performed without centralized control.

6. Addictiveness. Operations of IoV are adaptive and must adapt to either internal/external changes in the IoV. 
Complex agent-based applications using MASs often require high computing capabilities and collected data devices. Therefore, IoV infrastructure offers an ideal paradigm for MASs based systems because of its interconnected things/objects using communication protocols. The architecture of IoV is dynamic, which allows the system components to be reconfigured when it is needed at any time. It is worthy to say that the first four features mentioned above refer to the structural aspects of IoV, while the last other two features are used for functional purposes. For example, information agents in IoV can be employed for the processing of the information. Information agents are responsible for acquiring real time sensor data to process it and to obtain the required for the decision-making process. We believe that agent technology can help in developing IoV technology. The AOSE (agentoriented software engineering) paradigm is considered as a well-suited paradigm for developing complex software platforms in dynamic and distributed environments $[11,12]$. Thus AOSE will be a promising approach in developing IoV. Also, there are other middleware paradigms that are presented in [13-17] to support execution, deployment, and maintenance of IoT applications. Therefore, agent technologies offer several features to develop agent-based IoV applications as the following:

- middleware for IoV can be achieved by developing a multi-agent application;

- the integration of agent technologies into existing Vehicular Ad hoc Networks applications;

- multi-agent platforms can be extended with capabilities for IoV.

However, there is a significant number of MASs research of agent-based middleware; a major challenge still requires further research to enhance self-capabilities so that IoV can independently satisfy its goals regardless the environmental changes.

MASs implementation in IoV paradigm can be adapted based on the basic characteristics of intelligent agents such as proactivity, autonomy, negotiation, and learning as mentioned before. IoV is flexible to expand which is an important property for the execution of MASs applications that can adapt to the existing resources.

\section{Applications of Multi-Agent Systems on the Internet of Vehicles Context}

\section{A. Requirements}

As mentioned in earlier sections, IoV consists of many distributed components that can interact and that is heterogeneous in communication protocols and software interfaces. To effectively develop IoV, there are some requirements that are needed to be considered. Furthermore, the interconnection of agents through Internet-based network addresses critical issues that affect all network communications requirements, such as security, privacy and access control taking into account the sensitivity of the information exchanged by agents. Agents provide a degree of intelligence, control, and autonomy and can be integrated with IoV as in Figure 6 [18]. In CPSs, active agents could be the controller and passive agents, such as sensors, can provide inputs for the other agent. The interactions between an agent and its neighbors include negotiating about resource assignments and sharing of information. In the next, Figure 6 describes the different components and the architectural model of the integration of MASs and IoV. We start describing the physical components of different vehicles besides the key elements of the architecture. Then cyber entities technologies that can be used to interconnect the different parts of the IoV based on different MASs is described. The last part of this figure shows the interaction between the physical and cyber world with different stakeholders using cloud technology through the integration of this model.

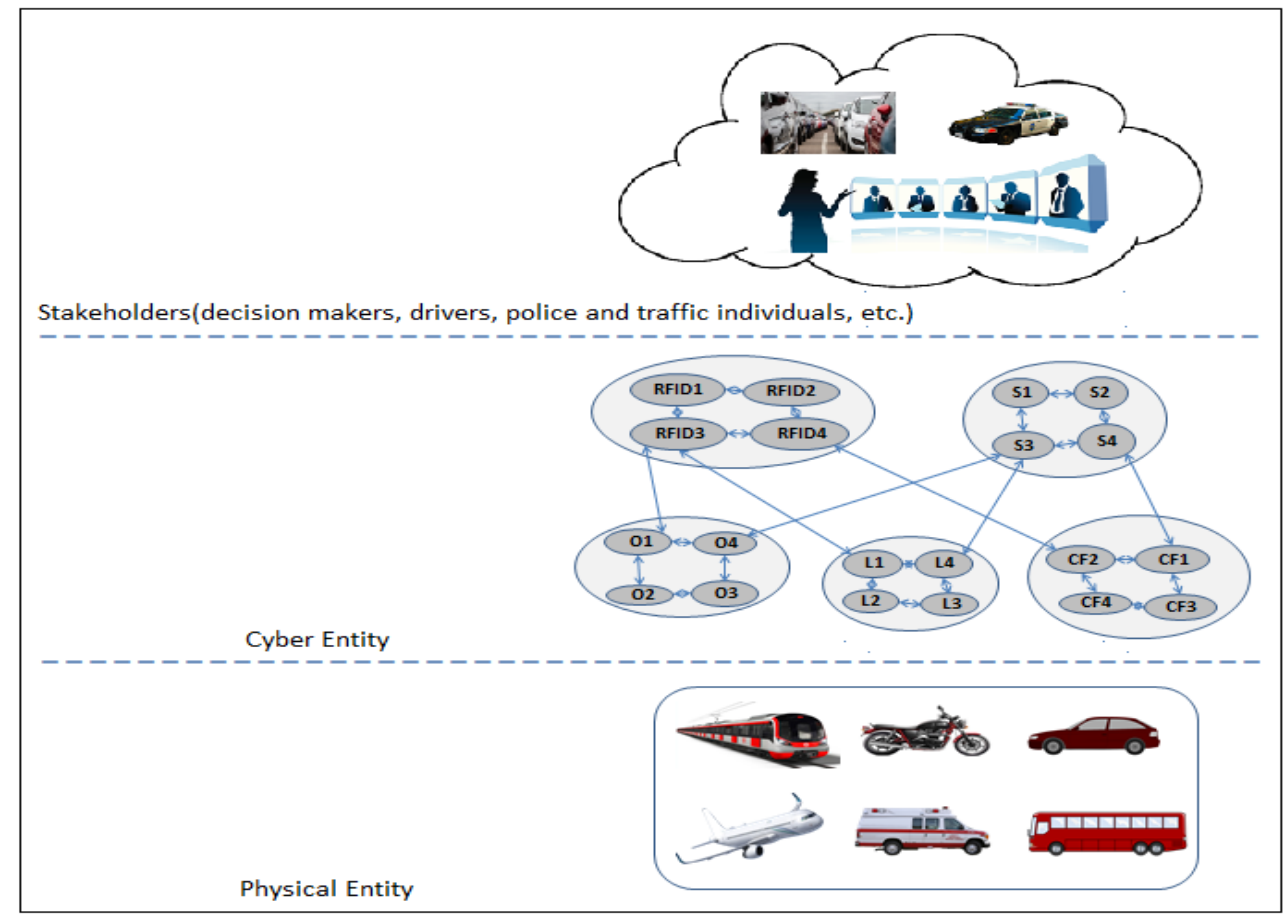

Fig. 6. The integration of MASs and IoV 


\section{B. Agents-based applications in IoV context}

In IoT platform, the things/objects are supposed not to be smart. There is no artificial intelligence software embedded in these things or objects to provide the ability of reasoning. But the IoT, on the whole, is intelligent. Also, the communication between things in IoT provides some intelligence because they perform smarter than the other things that are not connected to the Internet. MASs and IoT can be modeled in the smart cities to provide intelligence to the city services as proposed in [19], to offer efficient services and infrastructures with low-cost. Authors in [20] proposed a new solution based on intelligent software agents to support IoT and a new concept called the Agents of Things (AoT) is presented in this work. AoT is considered as an extension of IoT in which the things are embedded with self-reasoning intelligent software agents to provide value-added services to humans [21]. The AoT concept is presented to solve the problems such as the lack of intelligence in the things of the IoT systems. Therefore, AoT tries to support the IoT by embedding intelligent software agents in things. The main idea of AoT is that every object/thing has an intelligence capability. This intelligence enables the things to interact directly with other things. Moreover, it enables the things to interact with other systems. The work in [22] presents AoT concept's architecture and framework. In this proposed architecture authors proposed a hierarchical layered architecture based on the typical IoT architecture, but adding new layers to deal with the agent specific issues. In this scenario, there are many capabilities of the AoT in easing the issues associated with the traffic systems, speed monitoring, traffic light control, and road accidents. Therefore, the implementing of AoT extends the intelligence capability of the IoT to mitigate the effect of the IoT deficiencies and limitations.

In the proposed MobEyes model [23], vehicles use video cameras and sensors to record all events around the car accidents while driving. Thus, mobile and internet agents search the vehicular network for witnesses as part of their investigations.

There are other proposals related to IoT and agents in traffic context as in [24], where distributed agents are used in IoT system to control traffic-light intersections. This system uses a self-organized approach to ensure that the optimization of traffic-light in crossroads, using environmental data from IoT-like devices. Although this architecture defines local clusters of agents, this system is connected to the Internet to interconnect the local systems.

The interconnection of agents through an Internet-based network impose the protection of these systems, not only because of privacy issues but also because of the threat that an attack represents over infrastructures that manage services like urban transport or energy delivery. To solve such security issues, authors in [25] have shown a solution to apply a scheme that unifies access control systems between intelligent agents, IoT devices, and hybrid elements. This scheme used access control policies independently of the nature of the entities that interact in an IoT environment. This method allows an easy migration of the policy concept of agent communication and the management of different roles and permissions in the decision-making process during agent negotiation. This process implies an improvement in the access control management system. Also, authors in [26] presented the application of User-Managed Access (UMA) to provide a unified access control scheme for a heterogeneous hybrid architecture of IoT devices and intelligent agents used in traffic-light control. With this approach, the authors consider that they can employ the Internet to interconnect agents and devices while the security is not reduced and the environment can still take advantage of intelligent agents.

The authors in [26] proposed a multi-agent-based architecture to overcome such challenges of the Web of Things. This multiagent-based architecture can be embedded on real world thing. This architecture contains main classes such as DFA (Data Filtering Agent), CGA (Content Generation Agent), NCA (Networking and Communication Agent), and STPA (Security, Trust, and Privacy Agent). The goal of agent DFA is to process and analyze the data collected by local sensing devices, but also the data received from neighboring devices. The CGA will then be able to create some elementary services which will be published. The agent NCA uses appropriate communication protocols, such as 6LowPAN, Zigbee, WiFi, as well as appropriate communication pathways to respond and convey a requested service by a tier. The operation of the real world thing is carried out according to a specific security, trust, and privacy rules handled by the STPA [28]. Also, authors shared their vision on the Intelligent Web of Things (IWoT) and Social WoT through the use of MAS paradigm. The authors show the potential of their ideas presenting an application scenario, namely the intelligent Web of Vehicles.

Table I presents various applications in IoV context.

Table 1. IoV Applications

\begin{tabular}{|l|c|c|c|c|c|c|}
\hline Applications & {$[20]$} & {$[22]$} & {$[24]$} & {$[25]$} & {$[26]$} & {$[27]$} \\
\hline Urban monitoring & & & & & & $\mathrm{X}$ \\
\hline Road accidents & $\mathrm{X}$ & $\mathrm{X}$ & & & & \\
\hline Vehicle speed monitoring & $\mathrm{X}$ & & $\mathrm{X}$ & $\mathrm{X}$ & & \\
\hline Traffic light control & & & & & $\mathrm{X}$ & \\
\hline Avoid unexpected traffic jams & & & & & $\mathrm{X}$ & \\
\hline Information exchange & & & $\mathrm{X}$ & $\mathrm{X}$ & $\mathrm{X}$ & \\
\hline Security methods (considerations) & & & & & $\mathrm{x}$ & \\
\hline Freight transportation (smart logistics) & & & & \\
\hline
\end{tabular}




\section{The Proposed Model Evaluation, Challenges and Future Work}

In this section, we present the evaluation criteria for the proposed model. In addition to the challenges of this integration of MAS and IoV have been addressed and explained.

\section{A. Evaluation criteria}

The success of IoV relies on efficient vehicle-to-infrastructure (V2I) communications or vehicle-to-vehicle (V2V) communications to disseminate sensing and control data. Among all existing communication standards, the IEEE 802.15.4 standard, which comprises a simple physical (PHY) layer and an energy-efficient medium access control, is a promising candidate for IoV networks. Therefore, models for IEEE 802.15.4 are needed. The V2I communication allows vehicles to access communication services provided by the road side units (RSU). The RSU wire-line connected to the core network sends vehicle's data to the Internet. On the other hand, the $\mathrm{V} 2 \mathrm{~V}$ communication allows vehicles in the vicinity to exchange information via direct wireless links, thus reducing the latency and the traffic injected to the infrastructure network.

The location distance between the first vehicle and the second vehicle in this model need to be estimated in order to achieve high performance in accuracy and time efficiency. Because of the large overhead incurred for route discovery and route maintenance for highly mobile ordinated vehicles, only a few of the existing routing protocols for inter-vehicle networks are able to handle the requirements of safety applications. An important group of routing protocols for ad-hoc networks is based on topology, and needs the establishment of an end-to-end path between the source and the Because of the large overhead incurred for route discovery and route maintenance for highly mobile ordinated vehicles, only a few of the existing routing protocols for inter-vehicle networks are able to handle the requirements of safety applications. An important group of routing protocols for ad-hoc networks is based on topology, and needs the establishment of an end-to-end path between the source and the destination before sending any data package.

Based on the analysis of IoV and MASs with focusing on their strengths and features, we think that the performance of the proposed model will be improved for the field of transportation based on the running time and accuracy metrics. Moreover, the real results of implementing this integrated model will show high level of accuracy and performance than the current systems.

\section{B. Challenges and future work}

1. Agent control environment. Each agent operates in a degree of uncertainty, the variability of resources, dynamic in its environment. Some agents have another strategy in which they are operating under a decentralized control. Therefore challenges such as uncertainty, incompleteness, and ambiguity should be addressed.

2. Security issues. Security of agents is considered as one of the critical problems in vehicle aspect.

3. Information sharing and social networking.

To improve the performance of vehicles in IoV, some issues such as data locality and data routing for moving objects should also be addressed. Recently, social networks can play a significant role in vehicular networks. The SIoT paradigm is a social network in which things can establish social relationships with other things. Researchers believe that the integrated model of IoV and social networks will be provided in the Social Internet of Vehicles (SIoV) model [29]. Moreover, SIoV will extend the Social Internet of Things (SIoT) [30, 31]. Some problems such as information discovery and network navigability in IoT can be potentially solved by the social relationships between things. Some advantages of social networks can be exploited in SIoV [32, 33] from the interaction among drivers [34], vehicles and passengers [35].

In the future, the use of agent technologies in SIoV could provide various benefits and solutions to new challenges posed by the interconnection between entities on different levels (vehicles, drivers, passengers). For instance, the Human-Agent Collectives (HAC) paradigm [38] depicts new sociotechnical systems that mix computers and humans. The main idea of HAC paradigm is incorporating information gathers, humans, and processors in systems which include different agents of software [37-38]. In the HAC paradigm, smart software agents and humans involve in flexible social relationships to satisfy its goals. In this scenario, different resources, expert's consultations, resources, and the collected data and information can incorporate to operate in a coordinated way. Therefore, such systems have the ability to display a great autonomy and being characterized as social could provide multiple benefits for SIoV.

\section{Conclusion}

Over time, industrial and academic researchers proposed many advanced technologies for the IoV. This paper focusses on giving an overview of the multi-agent technologies and their applications in IoV, one of the key members of IoT, and do not describe the details of the technologies. Therefore, we reviewed the key elements and related technologies of the adopting agent technology in IoV and presented some applications that can be deployed. Based on our survey, we can state that agent-based IoV is still in its infancy. But, there are some benefits of applying MASs in IoV such as flexibility (agents are flexible to accommodate varieties of services to facilitate) and adaptability (agents adapt to varied network conditions such as vehicle mobility, the occurrence of critical events, changes in road and weather conditions, etc.).

Intelligent things can benefit from the software agent. The physical characteristic of these things can be embedded to improve their intelligence, ambient awareness, adaptive communication, negotiation capabilities, and pro-activeness. MASs require reliable 
distributed infrastructures, and IoV paradigm requires intelligent software with flexible, dynamic, and autonomous behavior that will result in new Internet applications and services. We can conclude that adopting agent technologies in IoV are still in the beginning, but there is an interest of researchers and decision makers of the industry to develop this approach. Future researchers should also address the integrating of social aspects with agent features in the IoV context.

\section{Acknowledgement}

The authors would like to acknowledge the valuable comments and suggestions of reviewers, which have improved the quality of the paper.

\section{References}

[1] E. L. George. The U.S. Economy and Monetary Policy, Federal Reserve Bank of Kansas City. [Online]. Available: http://www.kc.frb.org/publicat/speeches/2013-George-KansasCity-CentralExchange-01-10.pdf, 2013.

[2] U.S. Department of Transportation. Freight Transportation: Global Highlights. Retrieved from ww.rita.dot.gov: http://www.rita.dot.gov/bts/sites/rita.dot.gov.bts/files/publications/freight_transportation/pdf/entire.pdf,2010.

[3] M. Luck, P. McBurney, and C. Preist, "A manifesto for agent technology: Towards next generation computing," Auton. Agent Multi Agent Syst., vol. 9, no. 3, pp. 203-252, 2004.

[4] G. Fortino, "Agents Meet the IoT: Toward Ecosystems of Networked Smart Objects," in IEEE Systems, Man, and Cybernetics Magazine, vol. 2, no. 2, pp. 43-47, , doi: 10.1109/MSMC.2016.2557483, Available at http://ieeexplore.ieee.org/stamp/stamp.jsp?tp=\&arnumber=7549160\&isnumber=7549153, April 2016.

[5] A. Rogers, D. Corkill, and N. R. Jennings, “Agent technologies for sensor networks,” IEEE Intell. Syst., vol. 24, no. 2, pp. 13-17, 2009.

[6] G. Fortino, A. Guerrieri, W. Russo, “Agent-oriented smart objects development," in Proc. IEEE 16th Int. Conf. Computer Supported Cooperative Work in Design (CSCWD), pp. 907-912, 2012.

[7] S. Eberle, Adaptive internet integration of field bus systems, IEEE Trans. Ind. Inform. 3, 12-20, http://dx.doi.org/10.1109/ TII.2006.890525, 2007.

[8] A. Bechini, F. Marcelloni, A. Segatori, Low-effort support to efficient urban parking in a smart city perspective, in: S. Gaglio, G. Lo Re (Eds.), Advances onto the Internet of Things, Springer International Publishing, http://dx.doi.org/10.1007/978-3319-03992-3_17, 2014, pp. 233-252.

[9] K, P. Sycara: Multiagent systems. AI magazine 19(2), 79, 1998.

[10] C. Shanzhi, X. Hui, L. Dake, H. Bo, and W. Hucheng, A vision of IoT: Applications, Challenges, and Opportunities With China Perspective, IEEE INTERNET OF THINGS JOURNAL, VOL. 1, NO. 4, AUGUST, 2014.

[11] N. Jennings. On agent-based software engineering. Artificial intelligence. 31;117(2):277-96, 2000.

[12] N.R. Jennings and M. Wooldridge, "Agent-Oriented Software Engineering," in Handbook of Agent Technology, Bradshaw, J., Ed.: AAAI/MIT Press, 2001.

[13] K. Henricksen and R. Robinson, A survey of middleware for sensor networks: state-of-the art and future directions. Proceedings of the First International Workshop on Middleware for Sensor Networks (MidSens), Melbourne, Australia, 28 November, pp. 60-65. ACM, 2006.

[14] M. Molla, M and I. Ahamed, S., A survey of middleware for sensor network and challenges. ICPP Workshops, Columbus, Ohio, USA, 14-18 August, pp. 223-228. IEEE Computer Society, 2006.

[15] K. Lingaraj, R. Biradar, V. Patil, A Survey on Middleware Challenges and Approaches for Wireless Sensor Networks. IEEE International Conference on Computational Intelligence and Communication Networks (CICN) 2015 Dec 12 (pp. 56-60), 2015.

[16] I. Ungureanu, C. Nicoleta Gaitan, and G. Vasile Gheorghita, "A Middleware Based Architecture for the Industrial Internet of Things," KSII Transactions on Internet and Information Systems, vol. 10, no. 7, pp. 2874-2891, DOI: 10.3837/tiis.2016.07.001, 2016.

[17] Y. Kim, Y. Hong, D. Kim, Integrated Open Middleware for Interworking Sensor and Actuator Networks. Advanced Science Letters. 1;21(3):337-40, 2015.

[18] A. Kazi Masudul, S. Mukesh, E. Abdulmotaleb, Toward social Internet of Vehicles: Concept, Architecture, and Applications, Digital Object Identifier 10.1109/ACCESS.2015.2416657, 2015.

[19] M. Roscia, M. Longo, G. Lazaroiu. Smart city by multi-agent systems. InRenewable Energy Research and Applications (ICRERA), 2013 International Conference on 2013 Oct 20 (pp. 371-376), 2013.

[20] M. Mzahm, Anas., A. Mohd Sharifuddin, and T. Alicia YC, "Agents of Things (AoT): An intelligent operational concept of the Internet of Things (IoT)." In 2013 IEEE 13th International Conference on Intelligent Systems Design and Applications, pp. 159-164., 2013.

[21] M, Mzahm, Anas, A. Mohd Sharifuddin, T. Alicia YC, and A. Azhana. "Towards a Design Model for Things in Agents of Things." In Proceedings of the International Conference on Internet of things and Cloud Computing, p. 41. ACM, 2016.

[22] M, Mzahm, Anas, A. Mohd Sharifuddin, T. Alicia YC. "Enhancing the internet of things (IoT) via the concept of an agent of things (not)." Journal of Network and Innovative Computing 2, no.,101-110, 2014.

[23] U. Lee, E. Magistretti, B. Zhou, M. Gerla, P. Bellavista, and A. Corradi, "MobEyes: Smart Mobs for Urban Monitoring with a Vehicular Sensor Network,” IEEE Communications Magazine, vol. 13(6), pp. 52 - 57, Oct. 2006. 
[24] C. Turcu Elena, V. Gheorghiţă Găitan, and C. Octavian Turcu. "An internet of things-based distributed intelligent system with self-optimization for controlling traffic-light intersections." In Applied and Theoretical Electricity (ICATE), 2012 International Conference on, pp. 1-5. IEEE, 2012.

[25]L., Rivera, Diego, Luis Cruz-Piris, German Lopez-Civera, Enrique de la Hoz, and Ivan Marsa-Maestre. "Applying a unified access control for IoT-based Intelligent Agent Systems." In 2015 IEEE 8th International Conference on Service-Oriented Computing and Applications (SOCA), pp. 247-251. IEEE, 2015.

[26] N. Jabeur, and H. Hedi. "From Intelligent Web of Things to Social Web of Things." Facta Universitatis, Series: Electronics and Energetics 29, no. 3 (2015): 367-38, 2015.

[27] U. Lee U, Magistretti E, Gerla M, Bellavista P, Liò P, Lee KW. Bio-inspired multi-agent collaboration for urban monitoring applications. InBio-Inspired Computing and Communication, (pp. 204-216). Springer Berlin Heidelberg, 2008.

[28] M., Nitti, Roberto Grau, Alessandro Floris, and Luigi Atzori. "On adding the social dimension to the internet of vehicles: Friendship and middleware." In Communications and Networking (BlackSeaCom), 2014 IEEE International Black Sea Conference on, pp. 134-138. IEEE, 2014.

[29] L. Atzori, A. Iera, G. Morabito, and M.Nitti, “The social internet of things (SIoT) - when social networks meet the internet of things: Concept, architecture, and network characterization,” Computer Networks, vol. 56, no. 16, pp. 3594-3608, Nov. 2012.

[30] K. Alam, Saini M, El Saddik A. Toward social internet of vehicles: Concept, architecture, and applications. IEEE Access, 3:343-57, 2015.

[31] A. Yasar, Mahmud N, Preuveneers D, Luyten K, Coninx K, Berbers Y. Where people and cars meet: social interactions to improve information sharing in large scale vehicular networks. InProceedings of the 2010 ACM Symposium on Applied Computing 2010 Mar 22 (pp. 1188-1194), ACM, 2010.

[32] A. M. Vegni and V. Loscrí, "A Survey on Vehicular Social Networks," in IEEE Communications Surveys \& Tutorials, vol. 17, no. 4, pp. 2397-2419, Fourth quarter, doi: 10.1109/COMST.2015.2453481, Available at http://ieeexplore.ieee.org/stamp/stamp.jsp?tp=\&arnumber=7151778\&isnumber=7331734,2015.

[33] T. Luan, Lu R, Shen X, Bai F. Social on the road: Enabling secure and efficient social networking on highways. IEEE Wireless Communications, 22(1):44-51, 2015.

[34] L. Maglaras, Al-Bayatti AH, He Y, Wagner I, Janicke H. Social internet of vehicles for smart cities. Journal of Sensor and Actuator Networks, 6;5 (1):3, 2016.

[35] V. Podobnik, Lovrek I. An agent-based platform for ad-hoc social networking. InKES International Symposium on Agent and Multi-Agent Systems: Technologies and Applications, (pp. 74-83). Springer Berlin Heidelberg, 2011.

[36] E. Kamar, Gal YK, Grosz BJ. Modeling information exchange opportunities for effective human-computer teamwork. Artificial Intelligence, 195:528-50, 2013.

[37] M. Ptacek, Podobnik V, Jezic G. Beyond the Internet of Things: The Social Networking of Machines. International Journal of Distributed Sensor Networks. 2016 Jun 1, 2016.

[38] N. Jennings., Moreau, L., Nicholson, D., Ramchurn, S., Roberts, S., Rodden, T., Rogers, A.: Human-agent collectives. Communications of the ACM 57-12, 80-88, doi:10.1145/2629559, 2014. 